\title{
MOUVEMENTS PROPRES DES ETOILES DE REPERE \\ DU CATALOGUE PHOTOGRAPHIQUE
}

\author{
R. BOUIGUE \\ Université de Toulouse, France
}

Résumé. On examine le problème de l'obtention des mouvements propres à partir des observations meridiennes effectuées dans différents Observatoires, en vue de la détermination de la précision que l'on peut attendre de ces résultats.

On montre, en outre, que ces écarts ne sont pas imputables à l'hétérogénéité des observations et que l'on ne pourra donc les réduire par la simple création d'un seul et même système d'observation. 